\title{
Positive Position Feedback Controllers for Reduction the Vibration of a Nonlinear Spring Pendulum
}

\author{
Y.A. Amer, A.T. EL-Sayed, F.T. El-Bahrawy \\ Department of Mathematics, Faculty of Science, Zagazig University, Zagazig, Egypt \\ yaser31270@yahoo.com \\ Department of Basic Sciences, Modern Academy for Engineering and Technology, Maadi, Egypt \\ ashraftaha211@yahoo.com \\ Department of Basic Sciences, Modern Academy for Engineering and Technology, Maadi, Egypt \\ f_t_m_33@yahoo.com
}

\section{ABSTRACT}

In this paper, the two positive position feedback controllers (PPF) are proposed to reduce the longitudinal and angular vibrations of the nonlinear spring pendulum system which simulated the ship roll motion. This described by a four-degreeof-freedom system (4-DOF) which subjected to the external excitation force at simultaneous primary and internal resonance case. The method of multiple scale perturbation technique (MSPT) is applied to study the approximate solution of the given system. The stability of the system is investigated near the resonance case applying the frequency-response equations. Numerically, the effects of different controller's parameter's on the basic system behavior are studied.

\section{Keywords}

Vibration control; Positive position feedback; Stability; simultaneous resonance and spring pendulum.

\section{Nomenclature}

$$
\begin{array}{cl}
c_{j}, \mu_{j}(j=1,2) & \begin{array}{l}
\text { The damping coefficients of the spring pendulum modes and the PPF controllers, } \\
\text { respectively. }
\end{array} \\
\omega_{1}, \omega_{2}, \omega_{3} \text { and } \omega_{4} & \begin{array}{l}
\text { The natural frequencies of the spring pendulum modes and PPF controllers, } \\
\text { respectively. }
\end{array} \\
\alpha_{1} \text { and } \alpha_{2} & \text { The non-linear parameters } \\
f_{j} & \text { The external forcing amplitudes of the main system } \\
\Omega_{j} & \text { The excitation frequencies of the main system } \\
\varepsilon & \text { A small perturbation parameter. } \\
x & \text { Longitudinal response of the spring-pendulum } \\
\varphi_{1} & \text { Angular response of the pendulum } \\
u & \text { Displacement of the first PPF controller. } \\
v & \text { Displacement of the second PPF controller. } \\
\gamma_{1}, \gamma_{2} & \text { Control signal gains. } \\
\lambda_{2} & \text { Feedback signal gains. }
\end{array}
$$$$
\text { The natural frequencies of the spring pendulum modes and PPF controllers, }
$$

\section{INTRODUCTION}

Roll motion is one of the most significant phenomenon of a ship in waves. Therefor a lot of projects mentioned that. The roll motion of a ship can be fixed by analyzing various forms of moments acting on the ship, functional and actual mass moments of inertia roll damping moment, restoring moment, wave excitation and other moments caused by other modes of ship motion. Otherwise, in roll motion equation, there are a substantial term must be in the equation which is the damping term, that because among the other terms it controls on the magnitude of the amplitude. Though, it is the most difficult parameter to estimate because of its complex nature. The reason for its complexity is because of the non-linear nature of roll, and also because of the difficulty indetermining the roll damping characteristics. 
Osama et al. [1] investigated how to use active and passive controller to control ship roll. There are many famous engineering systems must be focused on. The spring pendulum and its application (ship's roll) one of this modeling system which studied in [2, 3]. Song et al. [4] illustrated how to apply the harmonic balance method to examine the vibration reaction of the spring-mass-damper system with a parametrically excited pendulum joined to the mass. Also, they indicated that the area of unstable motion of this model gained from the third order approximations is to be fairly consistent with that obtained from numerical calculations. Ahamed et al. [5] ) used positive position feedback controller to display the dynamic compensation for control of a rotary wing unmanned aerial vehicle (UAV).

Eissa et al. [6-8] using different techniques of passive and active control to discuss the vibration reduction for various systems. The vibration and stability of the non-linear spring pendulum were discussed to simulate the ship roll motion. Furthermore, they investigated the influnces of the transverse absorber on the non-linear spring pendulum system. It is worth to notice that such study is limited to multi-parametric excitations for each degree-of freedom. Shan et al. [9] applied The Positive Position Feedback (PPF) controller for a flexible drilling considering different vibration modes in the control techniques used for linear mathematical model. Also they made a comparison between the PPF control and the algorithm of velocity feedback. El-Ganaini W.A in [10] makes suppression for the vibration amplitude of nonlinear dynamical system at primary resonance that using for positive position feedback controller. Kwak and Heo [11] showed the action of the PPF algorithm applied for a model of a solar panel.

This work is an extension to the previous work [6-8] by adjusts the spring pendulum system with PPF controllers submitted to external excitation. To resolve the response of the transformed system near the simultaneous primary and internal resonance, the method of MSPT is applied. The frequency response of the discussed system applied to discuss the worst simultaneous resonance. The effectiveness of the PPF controllers on the discussed system and the influence of the different parameters on the given model were studied numerically.

\section{Mathematical model}

The vibration motion of the nonlinear spring-pendulum system which simulates the ship roll motion presented on Fig. 1. The dynamical behavior of the supposed system described as a nonlinear ordinary differential equations written in the following form:

$$
\begin{aligned}
& \ddot{x}+2 \varepsilon c_{1} \dot{x}+\omega_{1}^{2} x+\alpha_{1} x^{2}+\varepsilon^{-1} \alpha_{2} x^{3}-(1+x) \dot{\varphi}^{2}+\omega_{2}^{2}(1-\cos \varphi)=\varepsilon^{2} f_{1} \cos \left(\Omega_{1} t\right)+\varepsilon \gamma_{1} u \\
& \ddot{u}+2 \varepsilon \mu_{1} \omega_{3} \dot{u}+\omega_{3}^{2} u=\varepsilon \lambda_{1} x \\
& (1+x)^{2} \ddot{\varphi}+2 \varepsilon c_{2} \dot{\varphi}+2(1+x) \dot{x} \dot{\varphi}+\omega_{2}^{2}(1+x) \sin \varphi=\varepsilon^{2} f_{2} \cos \left(\Omega_{2} t\right)+\varepsilon \gamma_{2} v
\end{aligned}
$$$$
\ddot{v}+2 \varepsilon \mu_{2} \omega_{4} \dot{v}+\omega_{4}^{2} v=\varepsilon \lambda_{2} \varphi
$$

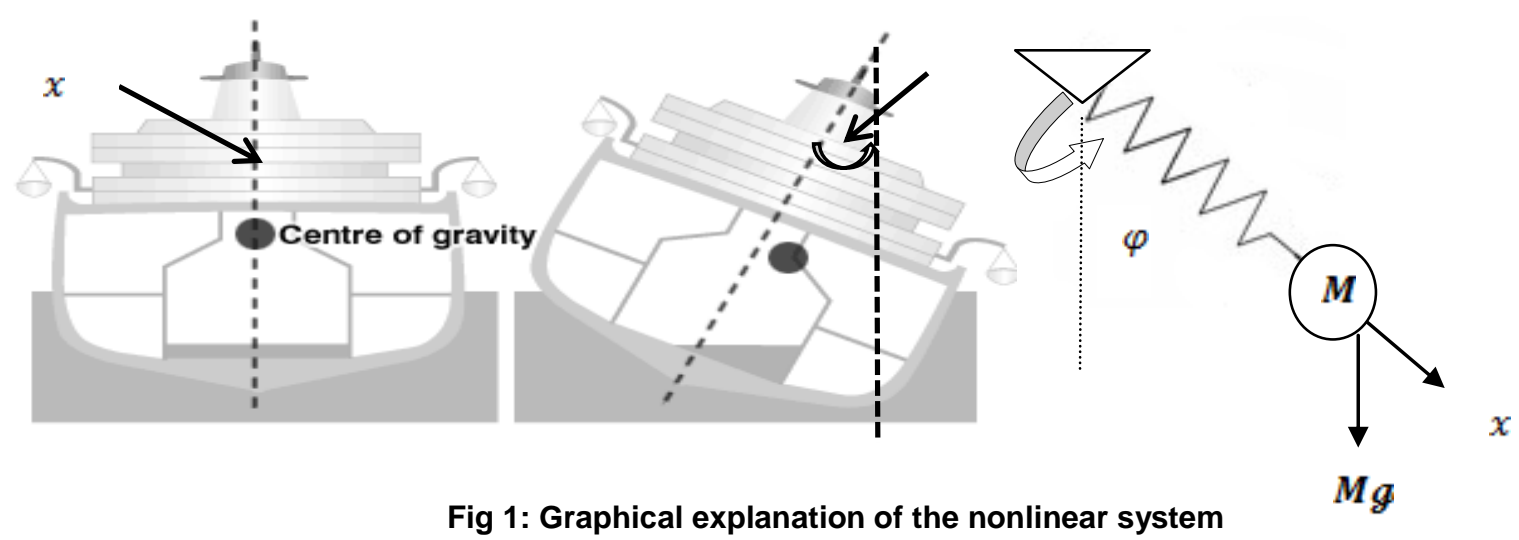

\section{Perturbation analysis}

Applying the multiple scales method to get the first-order approximate solutions for Eqs. (1-4) taking in consideration that, $\cos \varphi \cong 1-\frac{\varphi^{2}}{2 !}, \cos \varphi \cong \varphi-\frac{\varphi^{3}}{3 !}$ approximately as $\varphi<5^{\circ}$.

Seeking the solutions in the forms: 
$x(t ; \varepsilon)=\sum_{n=1}^{2} \varepsilon^{n} x_{n}\left(T_{0}, T_{1}\right)+o\left(\varepsilon^{3}\right)$

$\varphi(t ; \varepsilon)=\sum_{n=1}^{2} \varepsilon^{n} \varphi_{n}\left(T_{0}, T_{1}\right)+o\left(\varepsilon^{3}\right)$

$u(t ; \varepsilon)=\sum_{n=1}^{2} \varepsilon^{n} u_{n}\left(T_{0}, T_{1}\right)+o\left(\varepsilon^{3}\right)$

$v(t ; \varepsilon)=\sum_{n=1}^{2} \varepsilon^{n} v_{n}\left(T_{0}, T_{1}\right)+o\left(\varepsilon^{3}\right)$

Where $\varepsilon$ a small dimensionless bookkeeping perturbation parameter is $T_{0}=t, T_{1}=\varepsilon t$ are the fast and slow time scales, respectively. The time derivatives in terms of $T_{0}$ and $T_{1}$, will take the form

$\frac{d}{d t}=D_{0}+\varepsilon D_{1}$

$\frac{d^{2}}{d t^{2}}=D_{0}^{2}+2 \varepsilon D_{0} D_{1}$

Substituting from Eq. (5) and Eq. (6) into Eqs. (1-4) then we get:

$o(\varepsilon):$

$\left(D_{0}^{2}+\omega_{1}^{2}\right) x_{1}=0$

$\left(D_{0}^{2}+\omega_{2}^{2}\right) \varphi_{1}=0$

$\left(D_{0}^{2}+\omega_{3}^{2}\right) u_{1}=0$

$\left(D_{0}^{2}+\omega_{4}^{2}\right) v_{1}=0$

$o\left(\varepsilon^{2}\right):$

$\left(D_{0}^{2}+\omega_{2}^{2}\right) x_{2}=-2 D_{0} D_{1} x_{1}-2 c_{1} D_{0} x_{1}-\alpha_{1} x_{1}^{2}-\alpha_{2} x_{1}^{3}+\left(D_{0} \varphi_{1}\right)^{2}-\omega_{2}^{2} \frac{\varphi_{1}^{2}}{2}+f_{1} \cos \left(\Omega_{1} t\right)+\gamma_{1} u_{1}$

$\left(D_{0}^{2}+\omega_{2}^{2}\right) \varphi_{2}=-2 D_{0} D_{1} \varphi_{1}-2 x_{1} D_{0}^{2} \varphi_{1}-2 c_{2} D_{0} \varphi_{1}-2\left(D_{0} \varphi_{1}\right)\left(D_{0} x_{1}\right)-\omega_{2}^{2} x_{1} \varphi_{1}+f_{2} \cos \left(\Omega_{2} t\right)+\gamma_{2} v_{1}$

$\left(D_{0}^{2}+\omega_{3}^{2}\right) u_{2}=-2 D_{0} D_{1} u_{1}-2 \mu_{1} \omega_{3} D_{0} u_{1}+\lambda_{1} x_{1}$

$\left(D_{0}^{2}+\omega_{4}^{2}\right) v_{2}=-2 D_{0} D_{1} v_{1}-2 \mu_{2} \omega_{4} D_{0} v_{1}+\lambda_{2} \varphi_{1}$

The general solutions of the Eqs. (7-10) can be expressed as:

$x_{1}=A_{1} \exp \left(i \omega_{1} T_{0}\right)+c c$

$\varphi_{1}=A_{2} \exp \left(i \omega_{2} T_{0}\right)+c c$

$u_{1}=A_{3} \exp \left(i \omega_{3} T_{0}\right)+c c$

$v_{1}=A_{4} \exp \left(i \omega_{4} T_{0}\right)+c c$ 
Where $A_{m},(m=1,2, \ldots, 4)$ are complex functions in $T_{1}$ and $c c$ refers to the complex conjugate of the previous terms.

The first-order approximate solution will be obtained after substituting Eqs. (15)- (18) into Eqs. (11)- (14) and neglecting the secular term which written in the following form

$$
\begin{aligned}
& x_{2}=E_{1} \exp \left(2 i \omega_{1} T_{0}\right)+E_{2} \exp \left(3 i \omega_{1} T_{0}\right)+E_{3} \exp \left(2 i \omega_{2} T_{0}\right)+E_{6} \exp \left(i \Omega_{1} T_{0}\right)+E_{7} \exp \left(i \omega_{3} T_{0}\right)+E_{8}+c c \\
& \varphi_{2}=E_{9} \exp \left(i\left(\omega_{1}+\omega_{2}\right) T_{0}\right)+E_{10} \exp \left(i\left(\omega_{1}-\omega_{2}\right) T_{0}\right)+E_{11} \exp \left(i \Omega_{2} T_{0}\right)+E_{12} \exp \left(i \omega_{4} T_{0}\right)+c c \\
& u_{2}=E_{13} \exp \left(i \omega_{1} T_{0}\right)+c c \\
& v_{2}=E_{14} \exp \left(i \omega_{2} T_{0}\right)+c c
\end{aligned}
$$

Where the quantities $E_{n},\left(n=1,2, \ldots\right.$ are unknown functions in $T_{1}$ and $c c$ represent the complex conjugate of the previous terms.

Now we can get the analytical solution of Eqs. (1)-(4) by substituting Eqs. (15)-(22) into Eq. (5).

\section{Stability of the system}

The stability of the supposed model will be inspected from the first approximation at the simultaneous primary and internal resonance cases $\left(\Omega_{1}=\omega_{1}, \Omega_{2}=\omega_{2}, \omega_{3}=\omega_{1}\right.$ and $\left.\omega_{4}=\omega_{2}\right)$. Introducing the detuning parameters $\sigma_{1}, \sigma_{2}, \sigma_{3}$ and $\sigma_{4}$ according to:

$\Omega_{1}=\omega_{1}+\varepsilon \sigma_{1}, \Omega_{2}=\omega_{2}+\varepsilon \sigma_{2}, \omega_{3}=\omega_{1}+\varepsilon \sigma_{3}$

Substituting Eq. (23) into Eqs. (11-14) and neglecting the secular terms, leads to the solvability conditions for the first order approximation, hence the following differential equations are obtained:

$2 i \omega_{1} D_{1} A_{1}=-2 i c_{1} \omega_{1} A_{1}-3 \alpha_{2} A_{1}^{2} \bar{A}_{1}+\left[\frac{f_{1}}{2}\right] \exp \left(i \sigma_{1} T_{1}\right)+\left[\gamma_{1} A_{3}\right] \exp \left(i \sigma_{3} T_{1}\right)$

$2 i \omega_{2} D_{1} A_{2}=-2 i c_{2} \omega_{2} A_{2}+\left[\frac{f_{2}}{2}\right] \exp \left(i \sigma_{2} T_{1}\right)+\left[\gamma_{2} A_{4}\right] \exp \left(i \sigma_{4} T_{1}\right)$

$2 i \omega_{3} D_{1} A_{3}=\left[-2 i \mu_{1} \omega_{3}^{2} A_{3}\right]+\left[\lambda_{1} A_{1}\right] \exp \left(-i \sigma_{3} T_{1}\right)$

$2 i \omega_{4} D_{1} A_{4}=\left[-2 i \mu_{2} \omega_{4}^{2} A_{4}\right]+\left[\lambda_{2} A_{2}\right] \exp \left(-i \sigma_{4} T_{1}\right)$

To analyze Eqs. (24)-(27), put the following assumption:

$A_{m}=\frac{1}{2} a_{m} \exp \left(i \theta_{m}\right)$

Where $a_{m}$ and $\theta_{m}$ are real functions of $T_{1}$. Substituting from Eq. (28) into Eqs. (24-27) to get:

$$
\begin{aligned}
& \dot{a}_{1}=-c_{1} a_{1}+\frac{f_{1}}{2 \omega_{1}} \sin \beta_{1}+\frac{\gamma_{1} a_{3}}{2 \omega_{1}} \sin \beta_{3} \\
& a_{1} \dot{\theta}_{1}=\frac{3}{8 \omega_{1}} \alpha_{2} a_{1}^{3}-\frac{f_{1}}{2 \omega_{1}} \cos \beta_{1}-\frac{\gamma_{1} a_{3}}{2 \omega_{1}} \cos \beta_{3} \\
& \dot{a}_{2}=-c_{2} a_{2}+\frac{f_{2}}{2 \omega_{2}} \sin \beta_{2}+\frac{\gamma_{2} a_{4}}{2 \omega_{2}} \sin \beta_{4} \\
& a_{2} \dot{\theta}_{2}=-\frac{f_{2}}{2 \omega_{2}} \cos \beta_{2}-\frac{\gamma_{2} a_{4}}{2 \omega_{2}} \cos \beta_{4} \\
& \dot{a}_{3}=-\mu_{1} \omega_{3} a_{3}-\frac{\lambda_{1}}{2 \omega_{3}} a_{1} \sin \beta_{3}
\end{aligned}
$$


$a_{3} \dot{\theta}_{3}=-\frac{\lambda_{1} a_{1}}{2 \omega_{3}} \cos \beta_{3}$

$\dot{a}_{4}=-\mu_{2} \omega_{4} a_{4}-\frac{\lambda_{2}}{2 \omega_{4}} a_{2} \sin \beta_{4}$

$a_{4} \dot{\theta}_{4}=-\frac{\lambda_{2} a_{2}}{2 \omega_{4}} \cos \beta_{4}$

Where $\beta_{1}=\sigma_{1} T_{1}-\theta_{1}, \beta_{2}=\sigma_{2} T_{1}-\theta_{2}, \beta_{3}=\sigma_{3} T_{1}-\theta_{1}+\theta_{3}$ and $\beta_{4}=\sigma_{4} T_{1}-\theta_{2}+\theta_{4}$.

The steady- state solutions of the system according to the fixed points of Eqs. (29)- (36), will be analyzed if we put $\dot{a}_{m}=0 \quad$ and $\quad \dot{\beta}_{m}=0$. As well, the frequency response equations (FRE) for the practical case $\left(a_{1} \neq 0, a_{2} \neq 0, a_{3} \neq 0, a_{4} \neq 0\right)$ will be explained in follows:

$-c_{1} a_{1}+\frac{f_{1}}{2 \omega_{1}} \sin \beta_{1}+\frac{\gamma_{1} a_{3}}{2 \omega_{1}} \sin \beta_{3}=0$

$a_{1} \sigma_{1}-\frac{3}{8 \omega_{1}} \alpha_{2} a_{1}^{3}+\frac{f_{1}}{2 \omega_{1}} \cos \beta_{1}+\frac{\gamma_{1} a_{3}}{2 \omega_{1}} \cos \beta_{3}=0$

$-c_{2} a_{2}+\frac{f_{2}}{2 \omega_{2}} \sin \beta_{2}+\frac{\gamma_{2} a_{4}}{2 \omega_{2}} \sin \beta_{4}=0$

$a_{2} \sigma_{2}+\frac{f_{2}}{2 \omega_{2}} \cos \beta_{2}+\frac{\gamma_{2} a_{4}}{2 \omega_{2}} \cos \beta_{4}=0$

$-\mu_{1} \omega_{3} a_{3}-\frac{\lambda_{1}}{2 \omega_{3}} a_{1} \sin \beta_{3}=0$

$a_{3}\left(\sigma_{1}-\sigma_{3}\right)+\frac{\lambda_{1} a_{1}}{2 \omega_{3}} \cos \beta_{3}=0$

$-\mu_{2} \omega_{4} a_{4}-\frac{\lambda_{2}}{2 \omega_{4}} a_{2} \sin \beta_{4}=0$

$a_{4}\left(\sigma_{2}-\sigma_{4}\right)+\frac{\lambda_{2} a_{2}}{2 \omega_{4}} \cos \beta_{4}=0$

From Eqs. (41)-(44), we have:

$$
\begin{aligned}
& \sin \beta_{3}=\frac{-2 \mu_{1} \omega_{3}^{2} a_{3}}{\lambda_{1} a_{1}} \\
& \cos \beta_{3}=\frac{-2 a_{3} \omega_{3}\left(\sigma_{1}-\sigma_{3}\right)}{\lambda_{1} a_{1}} \\
& \sin \beta_{4}=\frac{-2 \mu_{2} \omega_{4}^{2} a_{4}}{\lambda_{2} a_{2}} \\
& \cos \beta_{4}=\frac{-2 a_{4} \omega_{4}\left(\sigma_{2}-\sigma_{4}\right)}{\lambda_{2} a_{2}}
\end{aligned}
$$


Squaring and adding Eqs. (45-46) and Eqs. (47-48), we get

$a_{1}^{2}=\frac{4 \omega_{3}^{2}}{\lambda_{1}^{2}}\left[\mu_{1}^{2} \omega_{3}^{2} a_{3}^{2}+a_{3}^{2}\left(\sigma_{1}-\sigma_{3}\right)^{2}\right]$
$a_{2}^{2}=\frac{4 \omega_{4}^{2}}{\lambda_{2}^{2}}\left[\mu_{2}^{2} \omega_{4}^{2} a_{4}^{2}+a_{4}^{2}\left(\sigma_{2}-\sigma_{4}\right)^{2}\right]$

Inserting Eqs.(45-46) in Eqs.(37-38), Eqs.(47-48) in Eqs.(39-40), squaring and adding the obtained results then we have ;

$$
\begin{aligned}
& {\left[-c_{1} a_{1}-\frac{\gamma_{1} a_{3}^{2} \mu_{1} \omega_{3}^{2}}{\omega_{1} \lambda_{1} a_{1}}\right]^{2}+\left[a_{1} \sigma_{1}-\frac{3}{8 \omega_{1}} \alpha_{2} a_{1}^{3}-\frac{\gamma_{1} a_{3}^{2} \omega_{3}\left(\sigma_{1}-\sigma_{3}\right)}{\omega_{1} \lambda_{1} a_{1}}\right]^{2}=\frac{f_{1}^{2}}{4 \omega_{1}^{2}}} \\
& {\left[-c_{2} a_{2}-\frac{\gamma_{2} a_{4}^{2} \mu_{2} \omega_{4}^{2}}{\omega_{2} \lambda_{2} a_{2}}\right]^{2}+\left[a_{2} \sigma_{2}-\frac{\gamma_{2} a_{4}^{2} \omega_{4}\left(\sigma_{2}-\sigma_{4}\right)}{\omega_{2} \lambda_{2} a_{2}}\right]^{2}=\frac{f_{2}^{2}}{4 \omega_{2}^{2}}}
\end{aligned}
$$

Where Eqs. (49-52) describe the frequency response.

The stability of the steady-state solution, will discussed in the following steps:

$a_{m}=a_{m 0}+a_{m 1}, \beta_{m}=\beta_{m 0}+\beta_{m 1}$

Where $a_{m 0}$ and $\beta_{m 0}$ are the solutions of Eqs. (29)-(36) and $a_{m 1}, \beta_{m 1}$ are perturbations which presupposed to be small compared with $a_{0}$ and $\beta_{0}$. Substituting Eq. (53) into Eqs. (29)-(36) and preserving the linear terms in $a_{m 1}$ and $\beta_{m 1}$, we obtain

$$
\begin{aligned}
& \dot{a}_{11}=\left[-c_{1}\right] a_{11}+\left[\frac{f_{1}}{2 \omega_{1}} \cos \left(\beta_{10}\right)\right] \beta_{11}+\left[\frac{\gamma_{1} a_{30}}{2 \omega_{1}} \cos \left(\beta_{30}\right)\right] \beta_{31}+\left[\frac{\gamma_{1}}{2 \omega_{1}} \sin \left(\beta_{30}\right)\right] a_{31} \\
& \dot{\beta}_{11}=\left[\frac{\sigma_{1}}{a_{10}}-\frac{9}{8 \omega_{1}} \alpha_{2} a_{10}\right] a_{11}-\left[\frac{f_{1}}{2 \omega_{1} a_{10}} \sin \left(\beta_{10}\right)\right] \beta_{11}-\left[\frac{\gamma_{1} a_{30}}{2 \omega_{1} a_{10}} \sin \left(\beta_{30}\right)\right] \beta_{31}+\left[\frac{\gamma_{1}}{2 \omega_{1} a_{10}} \cos \left(\beta_{30}\right)\right] a_{31} \\
& \dot{a}_{21}=\left[-c_{2}\right] a_{21}+\left[\frac{f_{2}}{2 \omega_{2}} \cos \left(\beta_{20}\right)\right] \beta_{21}+\left[\frac{\gamma_{2}}{2 \omega_{2}} \sin \left(\beta_{40}\right)\right] a_{41}+\left[\frac{\gamma_{2} a_{40}}{2 \omega_{2}} \cos \left(\beta_{40}\right)\right] \beta_{41} \\
& \dot{\beta}_{21}=\left[\frac{\sigma_{2}}{a_{20}}\right] a_{21}-\left[\frac{f_{2}}{2 \omega_{2} a_{20}} \sin \left(\beta_{20}\right)\right] \beta_{21}+\left[\frac{\gamma_{2}}{2 \omega_{2} a_{20}} \cos \left(\beta_{40}\right)\right] a_{41}-\left[\frac{\gamma_{2} a_{40}}{2 \omega_{2} a_{20}} \sin \left(\beta_{40}\right)\right] \beta_{41} \\
& \dot{a}_{31}=\left[-\mu_{1} \omega_{3}\right] a_{31}-\left[\frac{\lambda_{1}}{2 \omega_{3}} \sin \left(\beta_{30}\right)\right] a_{11}-\left[\frac{\lambda_{1}}{2 \omega_{3}} a_{10} \cos \left(\beta_{30}\right)\right] \beta_{31} \\
& \dot{\beta}_{31}=\left[\left(\frac{\sigma_{3}-\sigma_{1}}{a_{30}}\right)+\frac{\gamma_{1}}{2 \omega_{1} a_{10}} \cos \left(\beta_{30}\right)\right] a_{31}+\left[\frac{\sigma_{1}}{a_{10}}-\frac{9}{8 \omega_{1}} \alpha_{2} a_{10}-\frac{\lambda_{1}}{2 a_{30} \omega_{3}} \cos \beta_{30}\right] a_{11}-\left[\frac{f_{1}}{2 \omega_{1} a_{10}} \sin \left(\beta_{10}\right)\right] \beta_{11} \\
& +\left[\frac{\lambda_{1} a_{10}}{2 a_{30} \omega_{3}} \sin \beta_{30}-\frac{\gamma_{1} a_{30}}{2 \omega_{1} a_{10}} \sin \left(\beta_{30}\right)\right] \beta_{31}
\end{aligned}
$$




$$
\begin{aligned}
\dot{a}_{41} & =\left[-\mu_{2} \omega_{4}\right] a_{41}+\left[-\frac{\lambda_{2}}{2 \omega_{4}} a_{20} \cos \beta_{40}\right] \beta_{41}+\left[-\frac{\lambda_{2}}{2 \omega_{4}} \sin \beta_{40}\right] a_{21} \\
\dot{\beta}_{41} & =\left[\frac{\sigma_{2}}{a_{20}}-\frac{\lambda_{2}}{2 \omega_{4} a_{40}} \cos \beta_{40}\right] a_{21}-\left[\frac{f_{2}}{2 \omega_{2} a_{20}} \sin \left(\beta_{20}\right)\right] \beta_{21}+\left[\frac{\left(\sigma_{4}-\sigma_{2}\right)}{a_{40}}+\frac{\gamma_{2}}{2 \omega_{2} a_{20}} \cos \left(\beta_{40}\right)\right] a_{41} \\
& -\left[\frac{\gamma_{2} a_{40}}{2 \omega_{2} a_{20}} \sin \left(\beta_{40}\right)-\frac{\lambda_{2} a_{20}}{2 \omega_{4} a_{40}} \sin \beta_{40}\right] \beta_{41}
\end{aligned}
$$

Now let us put the eigenvalues of the above system of equations in the following form:

$$
\lambda^{8}+R_{1} \lambda^{7}+R_{2} \lambda^{6}+R_{3} \lambda^{5}+R_{4} \lambda^{4}+R_{5} \lambda^{3}+R_{6} \lambda^{2}+R_{7} \lambda+R_{8}=0
$$

Where $\left(R_{1}, R_{2}, \ldots, R_{8}\right)$ are functions of the parameters $\left(a_{1}, a_{2}, a_{3}, a_{4}, \omega_{1}, \omega_{2}, \omega_{3}, \omega_{4}, \sigma_{1}, \sigma_{2}, \sigma_{3}, \sigma_{4}, \sigma_{5}, \sigma_{6}, c_{1}, c_{2}, \mu_{1}, \mu_{2}\right.$, $\left.f_{1}, f_{2}, \alpha_{1}, \alpha_{2}, \beta_{1}, \beta_{2}, \beta_{3}, \beta_{4}, \gamma_{1}, \gamma_{2}, \lambda_{1}, \lambda_{2}\right)$. If and only if the real part of the eigenvalue, which obtained from eigen equation (62), is negative, then the periodic solution is stable; otherwise, it is unstable. The necessary and sufficient conditions for all the roots of Eq. (62) will be calculated according to the Routh-Hurwitz criterion to have negative real parts if and only if the determinant $D$ and all its principle minors are positive.

$$
D=\left|\begin{array}{cccccccc}
R_{1} & 1 & 0 & 0 & 0 & 0 & 0 & 0 \\
R_{3} & R_{2} & R_{1} & 1 & 0 & 0 & 0 & 0 \\
R_{5} & R_{4} & R_{3} & R_{2} & R_{1} & 1 & 0 & 0 \\
R_{7} & R_{6} & R_{5} & R_{4} & R_{3} & R_{2} & R_{1} & 1 \\
0 & R_{8} & R_{7} & R_{6} & R_{5} & R_{4} & R_{3} & R_{2} \\
0 & 0 & 0 & R_{8} & R_{7} & R_{6} & R_{5} & R_{4} \\
0 & 0 & 0 & 0 & 0 & R_{8} & R_{7} & R_{6} \\
0 & 0 & 0 & 0 & 0 & 0 & 0 & R_{8}
\end{array}\right|
$$

\section{Numerical Solutions}

The differential equations of the basic system with PPF controllers (1-4) at simultaneous primary and internal resonance cases $\left(\Omega_{1}=\omega_{1}, \quad \Omega_{2}=\omega_{2}, \omega_{4} \cong \omega_{2}\right.$, and $\omega_{3} \cong \omega_{1}$ ) were solved numerically applying Rung-Kutta fourth order method. The simulation results are achieved by using MATLAB 7.14 (R2012a) package (ode45).

The reaction of the phase-plane of the basic system without control at the primary resonance case in Fig.2 at the selected estimate of the equation parameters $\left(\Omega_{1}=\omega_{1}, \alpha_{1}=0.004, \alpha_{2}=0.006, f_{1}=0.005, f_{2}=0.004, \omega_{1}=5, \omega_{2}=4, c_{1}=0.04\right.$, and $c_{2}=0.015$, ) 

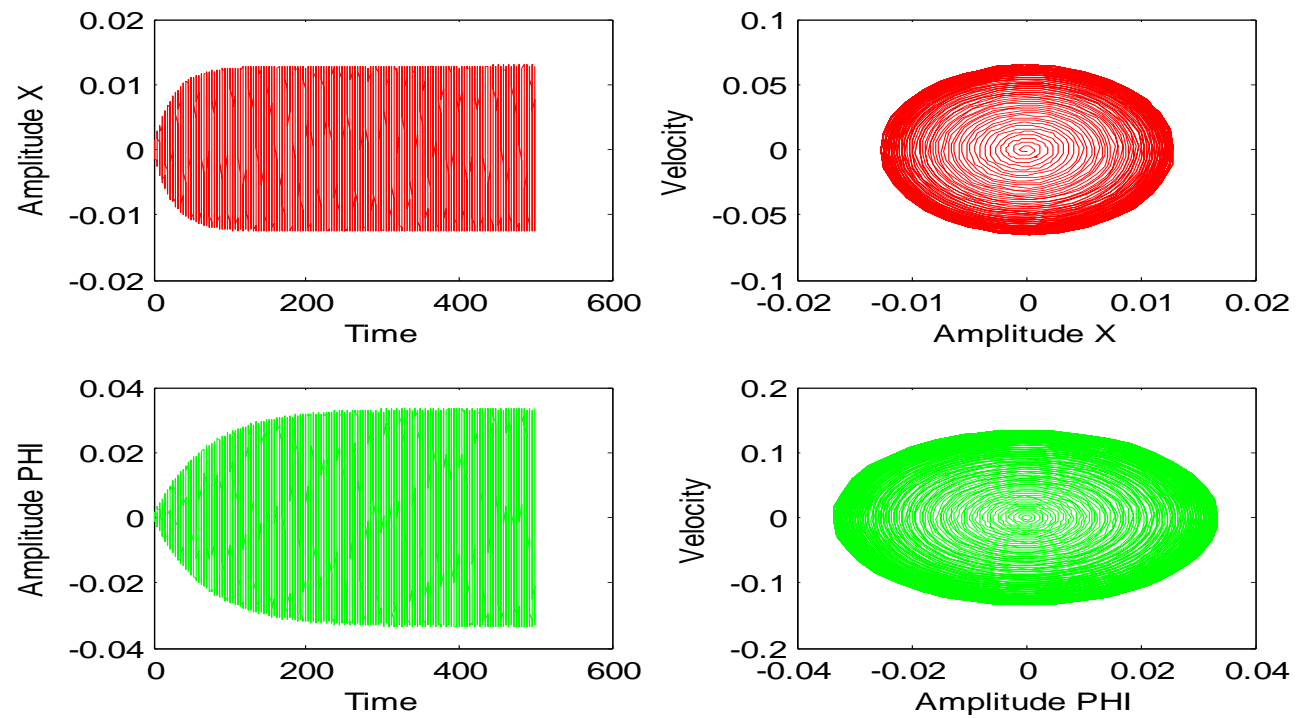

Fig 2: the basic system $(x, \varphi)$ without controllers at $\left(\Omega_{1}=\omega_{1}, \Omega_{2}=\omega_{2}\right)$

Fig. 3 proved that the steady state amplitudes of the basic system first mode $(x)$ and second mode $(\varphi)$ with PPF controllers are decreasing to about $99.92 \%$ and about $99.99 \%$ from its value without controllers, respectively. The steady state amplitude of the PPF controllers $(u)$ and $(v)$ is about 0.0005 and 0.000399 , respectively as seen in Fig. 3 . This means that the effectiveness of the controllers $E_{a}$ ( $E_{a}=$ steady-state amplitude of the main system without control $/$ steady-state amplitude of the main system with control) are about 1319 for $(x)$ and about 11795 for $(\varphi)$.
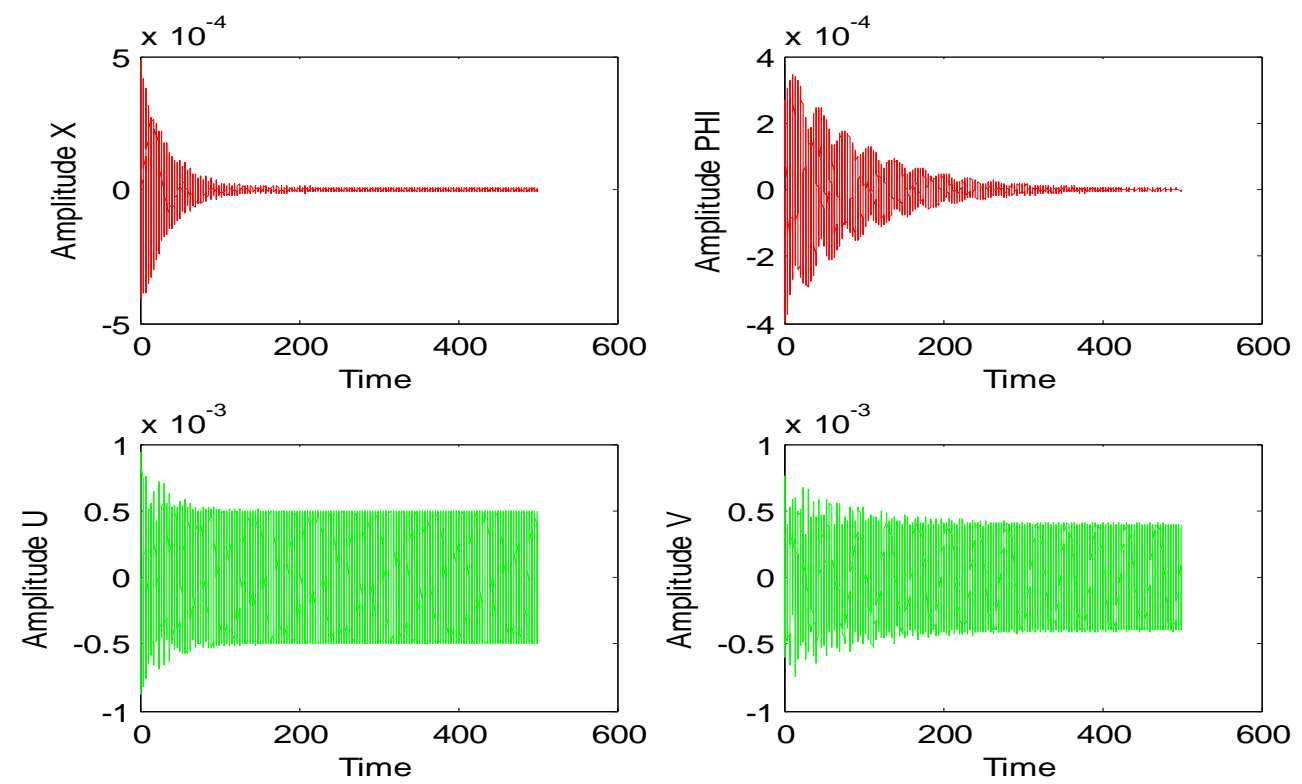

Fig 3: Response of the system with PPF controllers at simultaneous resonance case

$$
\left(\Omega_{1} \cong \omega_{1}, \Omega_{2} \cong \omega_{2}, \omega_{3} \cong \omega_{1}, \omega_{4} \cong \omega_{2}\right)
$$

\subsection{Frequency response curve with the detuning parameter $\left(\sigma_{1}\right)$}

In this section, the frequency response equations (49-51) of the basic system (first mode) and the PPF controller are explained numerically. Results are declared in graphical forms as steady-state amplitudes $\left(a_{1}\right.$ and $\left.a_{3}\right)$ against the detuning parameters $\sigma_{1}$ for both the basic system and the PPF controller, respectively as seen in Fig. 4.

Fig.5 (a, b) presented that for the large value of $\omega_{1}$, the band width for the steady state amplitudes of the basic system $\left(a_{1}\right)$ and PPF control $\left(a_{3}\right)$ are decrease but for small amount of $\omega_{1}$ the band width increased. 
Fig $6(\mathrm{a}, \mathrm{b})$ and $7(\mathrm{a}, \mathrm{b})$ illustrate that the peak amplitudes for the basic system $\left(a_{1}\right)$ and the PPF control ( $\left.a_{3}\right)$ are monotonic decreasing function to the damping coefficients $c_{1}$ and $\mu_{1}$.

It is clear that, when the external excitation force $f_{1}$ is increase the steady state amplitudes for the main system $\left(a_{1}\right)$ and the controller $\left(a_{3}\right)$ are increases as seen in Fig.8 $(\mathrm{a}, \mathrm{b})$.

Fig. $9(\mathrm{a}, \mathrm{b})$ show that for the large value of the feedback signal gain $\lambda_{1}$, the band width for the basic system amplitude $\left(a_{1}\right)$ and the controller amplitude $\left(a_{3}\right)$ are wider. Also, the controller amplitude $\left(a_{3}\right)$ is monotonic increasing function to $\lambda_{1}$ as presented in Fig.9 (b)

Moreover, Fig.10 (a) show that the band width for the main system's amplitude $\left(a_{1}\right)$ and the controller's amplitude $\left(a_{3}\right)$ are wider for the large value of $\gamma_{1}$, also in Fig. 10(b) the non-zero solution for the controller amplitude at $\sigma_{1}=0$ is increase.

Fig. 11(a, b) we see that the influence for increasing or decreasing values of the nonlinear parameter $\alpha_{2}$ in both the basic system $\left(a_{1}\right)$ and the controller $\left(a_{3}\right)$ are trivial due to the occurrence of saturation phenomena.

Fig. 12 shows the steady state amplitude of both the basic system $\left(a_{1}\right)$ and the controller $\left(a_{3}\right)$ for three distinct values of the internal detuning parameter $\sigma_{3}$. Here, Fig 12(a) show that for $\sigma_{3}=0.0$ the minimum basic system steady-state amplitude exists when $\sigma_{1}=0.0$, for $\sigma_{3}=0.5$ the minimum main system steady-state amplitude exists when $\sigma_{1}=0.5$ and for $\sigma_{3}=-0.5$ the minimum main system steady-state amplitude exists when $\sigma_{1}=-0.5$. Based on Fig. 12(a) the minimum main system steady-state amplitude exists when $\sigma_{1}=\sigma_{3}$
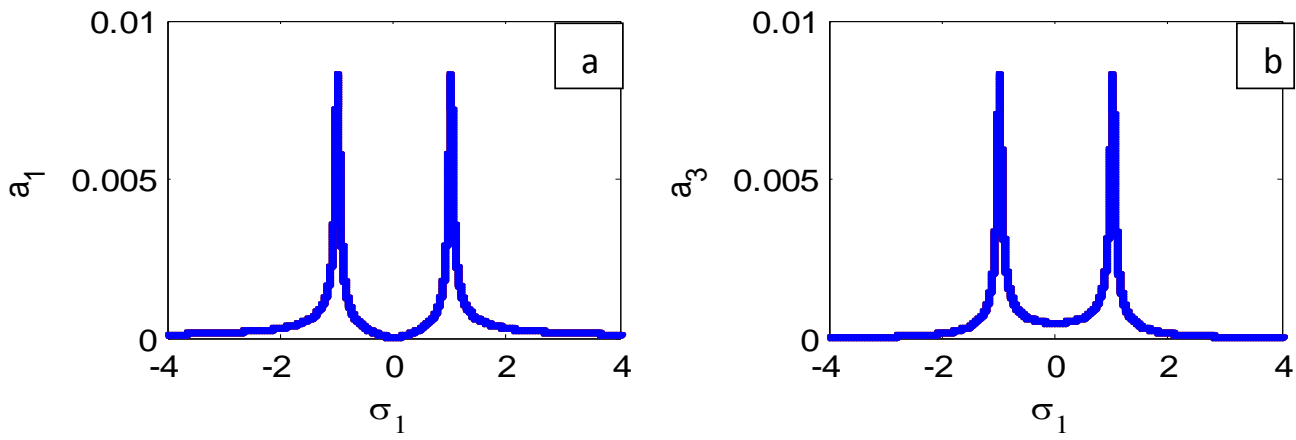

Fig 4: Frequency response curves of: (a) fist mode of the basic system $\left(a_{1}\right)$ and (b) the controller $\left(a_{3}\right)$
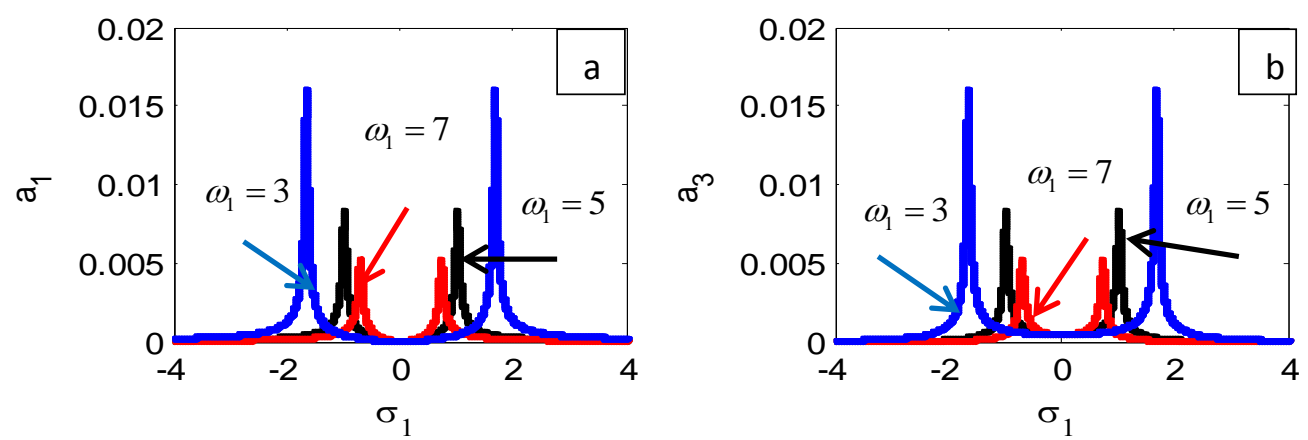

Fig 5: Influence of the natural frequency $\omega_{1}$ on (a) the basic system $\left(a_{1}\right)$ and (b) the controller $\left(a_{3}\right)$ 
Journal of Advances in Mathematics
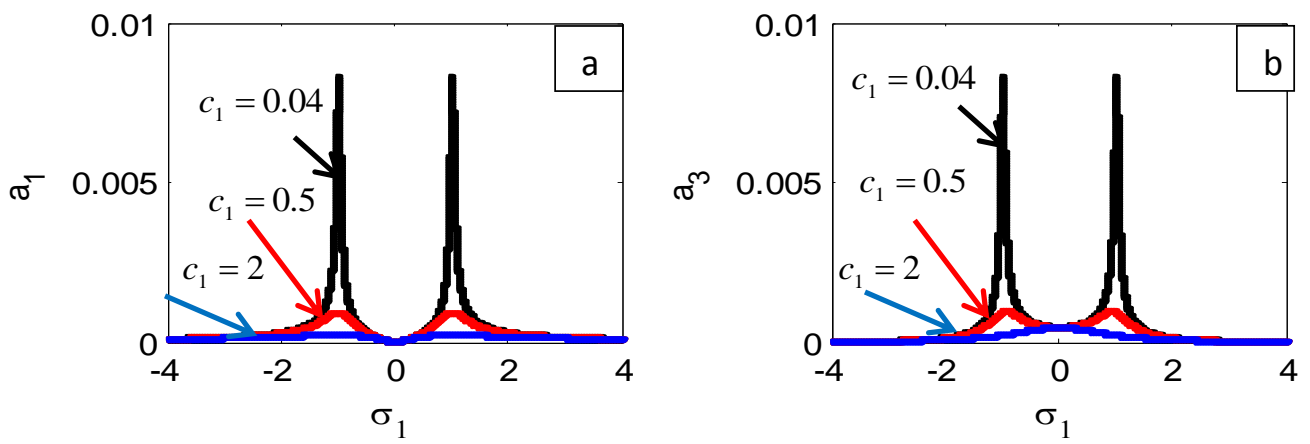

Fig 6:Influence of The damping coefficient $c_{1}$ on (a) the basic system $\left(a_{1}\right)$ and (b) the controller $\left(a_{3}\right)$
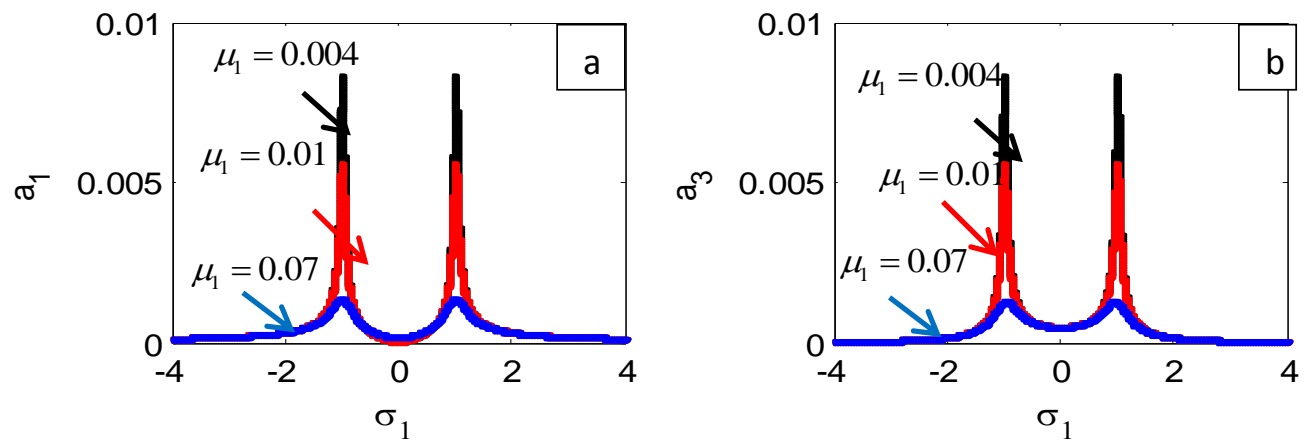

Fig 7:Influence of The damping coefficient $\mu_{1}$ on (a) the basic system $\left(a_{1}\right)$ and (b) the controller $\left(a_{3}\right)$
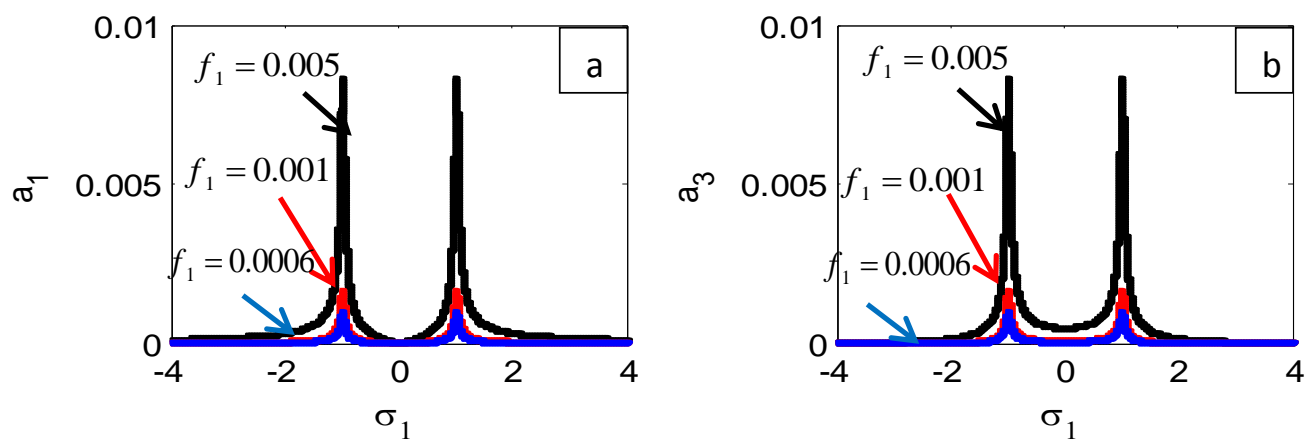

Fig 8:Influence of The external excitation force $f_{1}$ on (a) the basic system $\left(a_{1}\right)$ and (b) the controller $\left(a_{3}\right)$
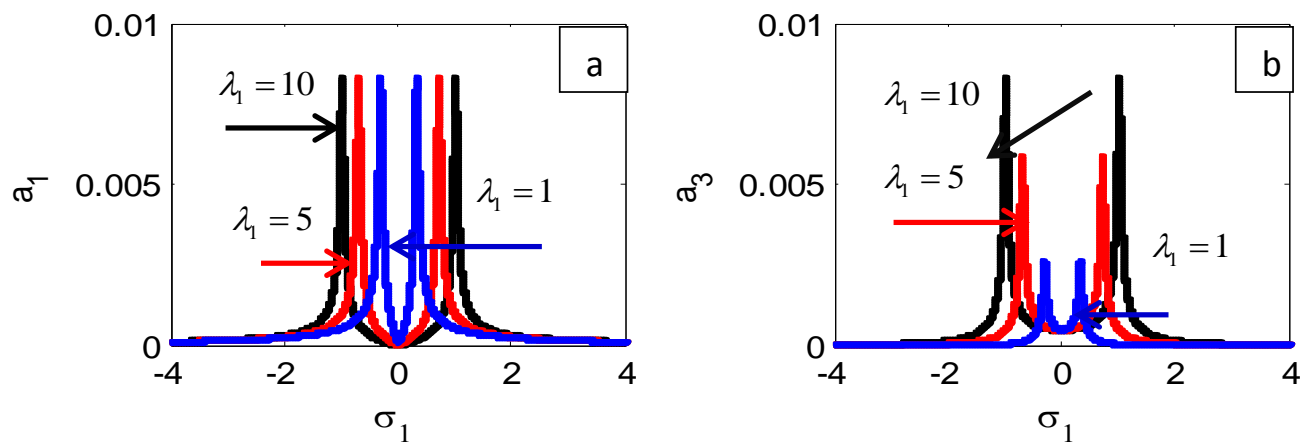

Fig 9:Influence of The varying the feedback signal gain $\lambda_{1}$ on (a) the basic system $\left(a_{1}\right)$ and (b) the controller $\left(a_{3}\right)$ 

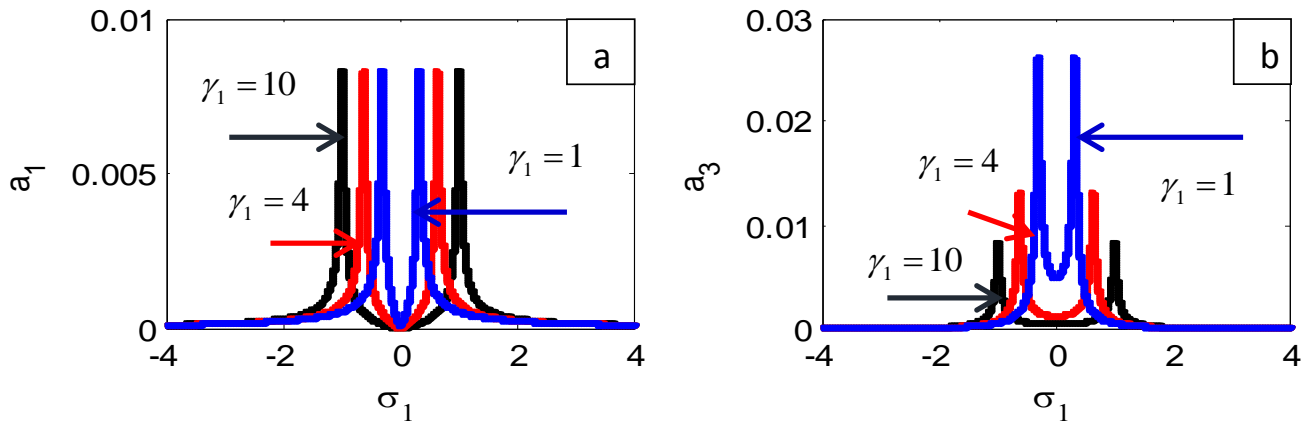

Fig 10:Influence of The control signal gain $\gamma_{1}$ on (a) the basic system $\left(a_{1}\right)$ and (b) the controller $\left(a_{3}\right)$
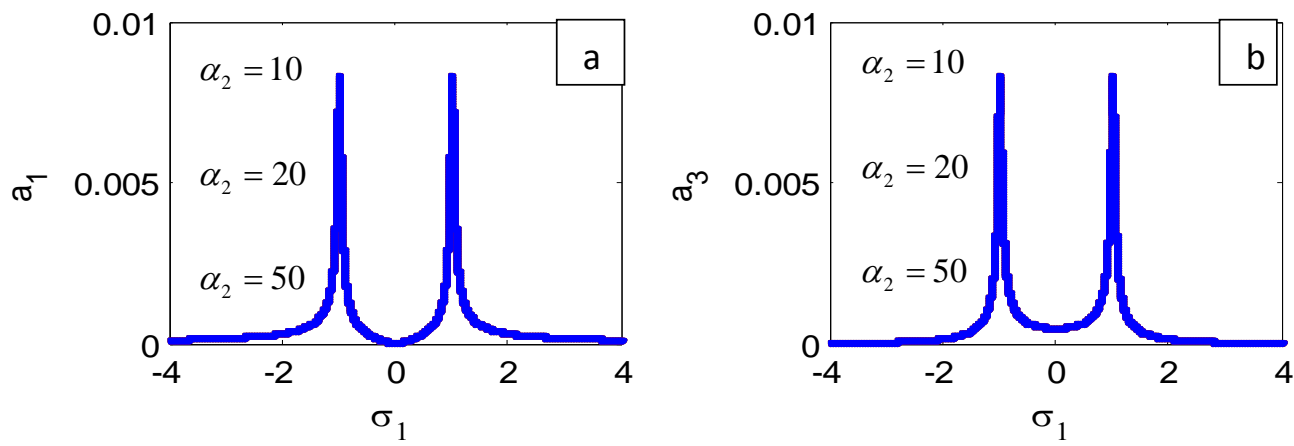

Fig 11:Influence of The nonlinear parameter $\alpha_{2}$ on (a) the basic system $\left(a_{1}\right)$ and (b) the controller $\left(a_{3}\right)$
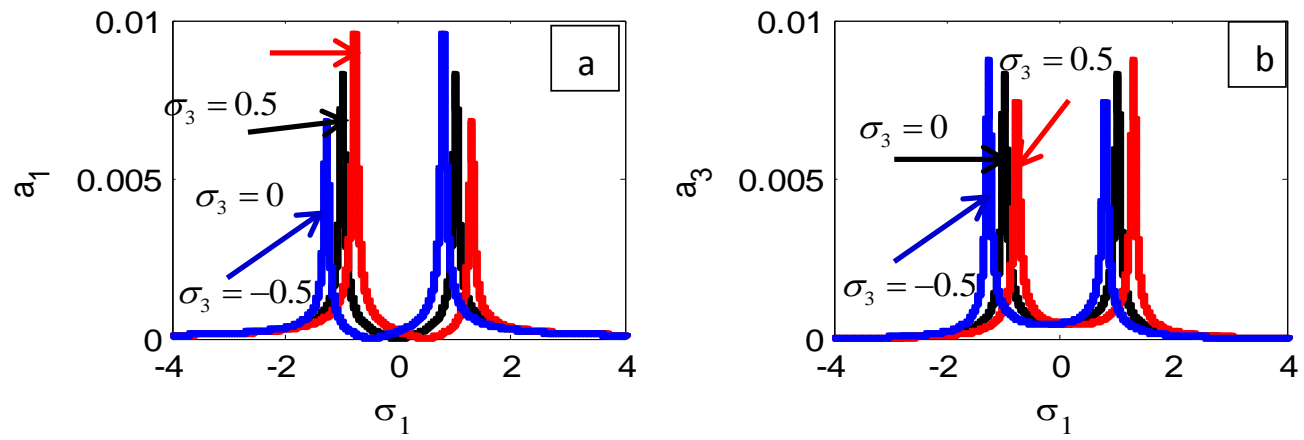

Fig 12:Influence of The detuning parameter $\sigma_{3}$ on (a) the basic system $\left(a_{1}\right)$ and (b) the controller $\left(a_{3}\right)$

\subsection{Frequency response curve with the detuning parameter $\left(\sigma_{2}\right)$}

Furthermore, the steady-state amplitudes for the second mode $\left(a_{2}\right)$ and $\operatorname{PPF}$ controller $\left(a_{4}\right)$ versus the variations of $\sigma_{2}$ are plotted as illustrated in Fig. 13.

Fig.14 (a, b) presented that for large value of $\omega_{2}$, the band width and the steady state amplitudes of the main system $\left(a_{2}\right)$ and PPF control $\left(a_{4}\right)$ are decrease.

Fig. $15(\mathrm{a}, \mathrm{b})$ and $16(\mathrm{a}, \mathrm{b})$ presented that the steady state amplitudes of the main system $\left(a_{2}\right)$ and PPF control ( $\left.a_{4}\right)$ are decreases when the damping coefficients $c_{2}$ and $\mu_{2}$ are increases.

Also, Fig. $17(\mathrm{a}, \mathrm{b})$ show the steady state amplitudes of the main system $\left(a_{2}\right)$ and PPF control $\left(a_{4}\right)$ are monotonic increasing function to the external excitation force $f_{2}$.

For the large value of $\lambda_{2}$ and $\gamma_{2}$, the band width for the steady state amplitudes of the main system $\left(a_{2}\right)$ and PPF control $\left(a_{4}\right)$ is wider as shown in Fig. 18(a, b) and Fig. 19(a, b). Also, the controller's amplitude is monotonic increasing function to $\lambda_{2}$ as illustrated in Fig.18 (b) and monotonic decreasing function to $\gamma_{2}$ as illustrated in Fig.19 (b). 
For three different values of the internal detuning parameter $\sigma_{4}$, the branches for the amplitudes of both the main system $\left(a_{2}\right)$ and the controller $\left(a_{4}\right)$ are increase and decrease as shown in Fig. 20(a, b). Here, Fig. 20(a) show that for $\sigma_{4}=0.0$, the minimum main system steady-state amplitude happens when $\sigma_{2}=0.0$, for $\sigma_{4}=0.2$ the minimum main system steadystate amplitude occurs when $\sigma_{2}=0.2$, and for $\sigma_{4}=0.7$ the minimum main system steady-state amplitude occurs when $\sigma_{2}=0.7$. Based on Fig. 20(a) the minimum main system steady-state amplitude occurs when $\sigma_{2}=\sigma_{4}$.
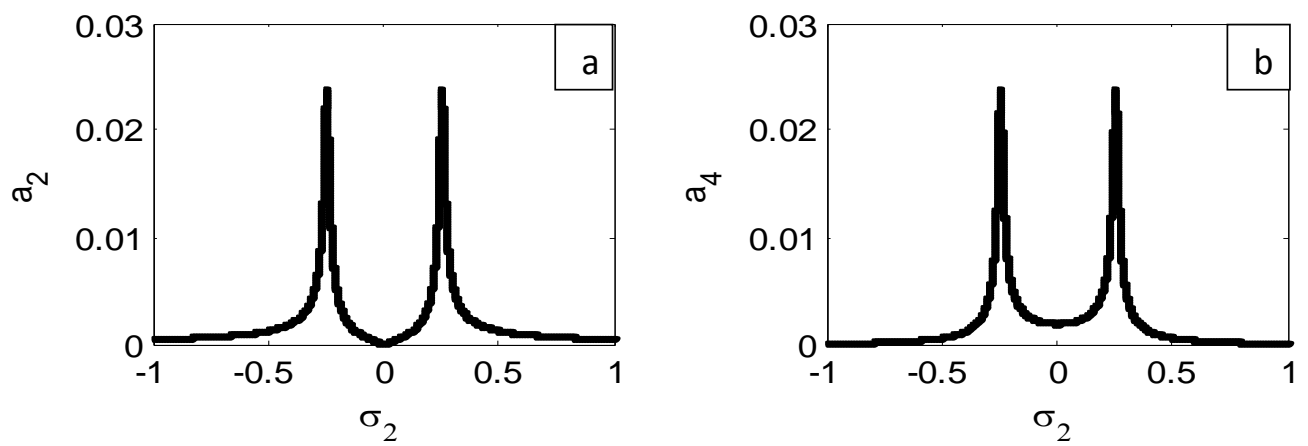

Fig 13: Frequency-response curves of on (a) second mode of the system $\left(a_{2}\right)$ and (b) PPF controller $\left(a_{4}\right)$
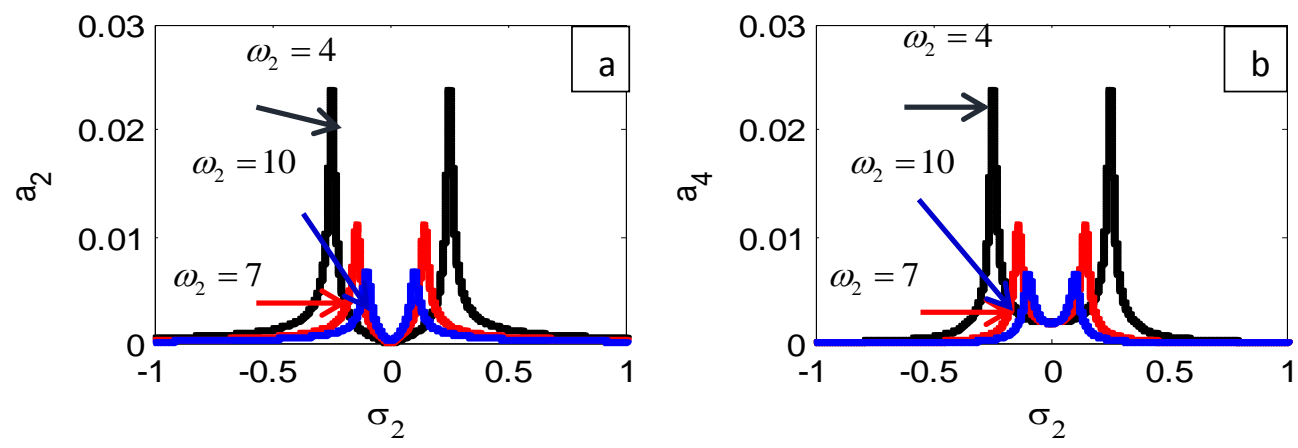

Fig 14: Influence of the natural frequency $\omega_{2}$ (a) second mode of the system $\left(a_{2}\right)$ and (b) PPF controller $\left(a_{4}\right)$
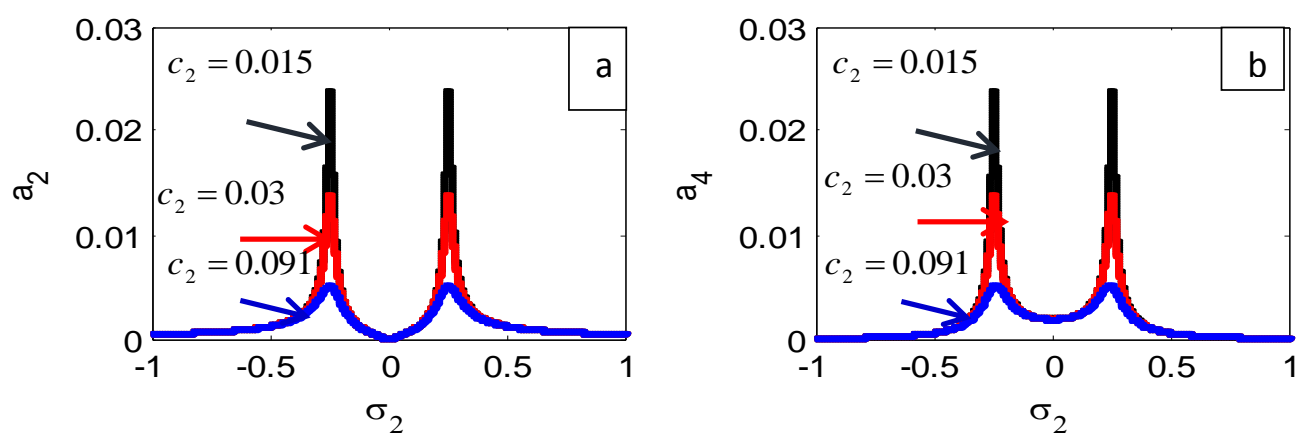

Fig 15: Influence of The damping coefficient $c_{2}$ (a) second mode of the system $\left(a_{2}\right)$ and (b) PPF controller $\left(a_{4}\right)$
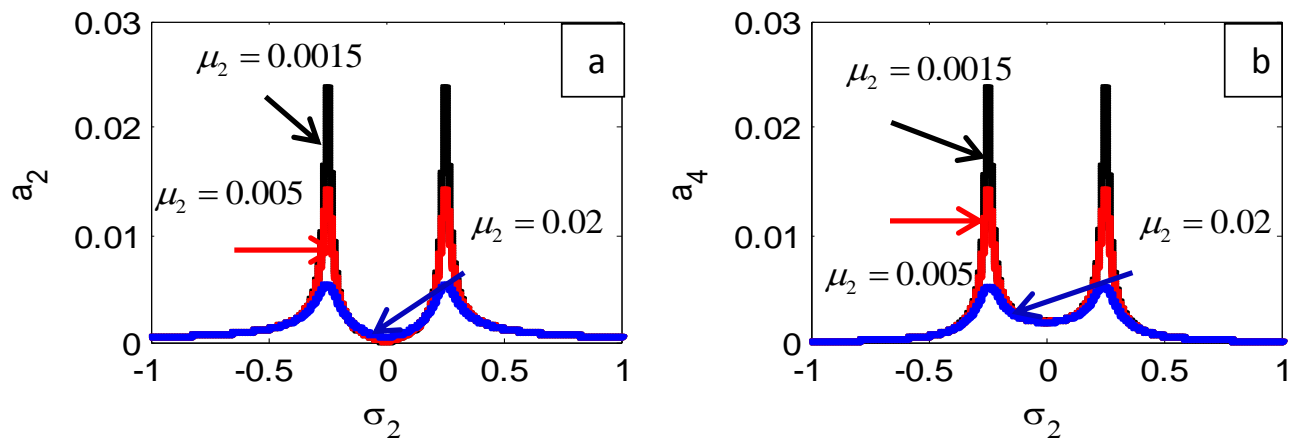
Journal of Advances in Mathematics

Fig 16: Influence of the damping coefficients $\mu_{2}$ (a) second mode of the system $\left(a_{2}\right)$ and (b) PPF controller $\left(a_{4}\right)$
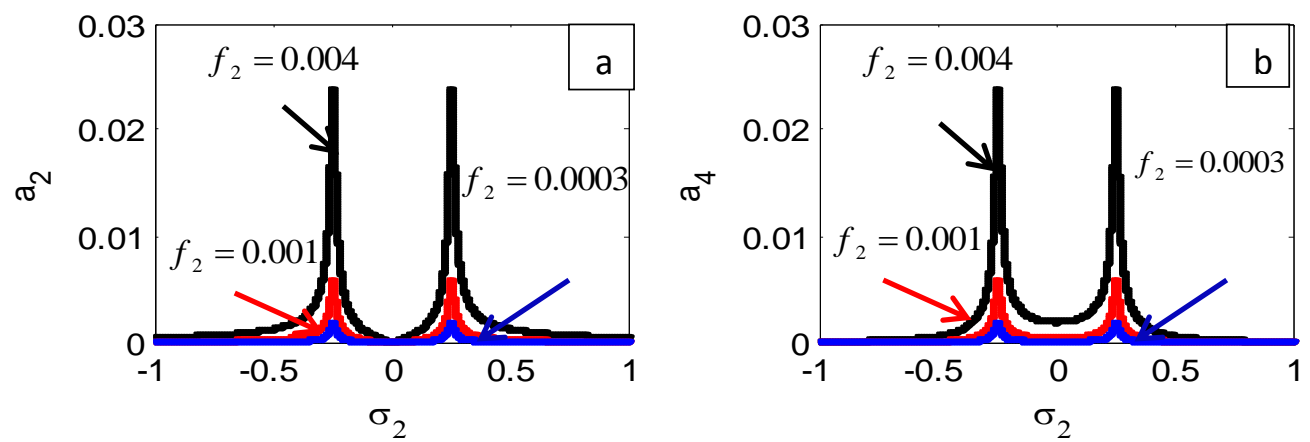

Fig 17: Influence of the external excitation force $f_{2}$ (a) second mode of the system $\left(a_{2}\right)$ and (b) PPF controller $\left(a_{4}\right)$
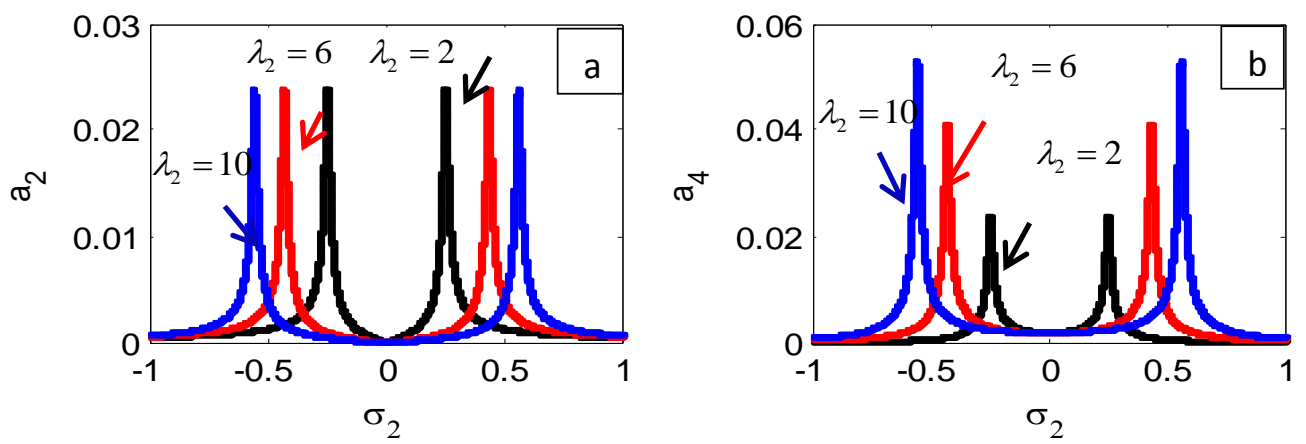

Fig 18: Influence of the feedback signal gain $\lambda_{2}$ (a) second mode of the system $\left(a_{2}\right)$ and (b) PPF controller $\left(a_{4}\right)$
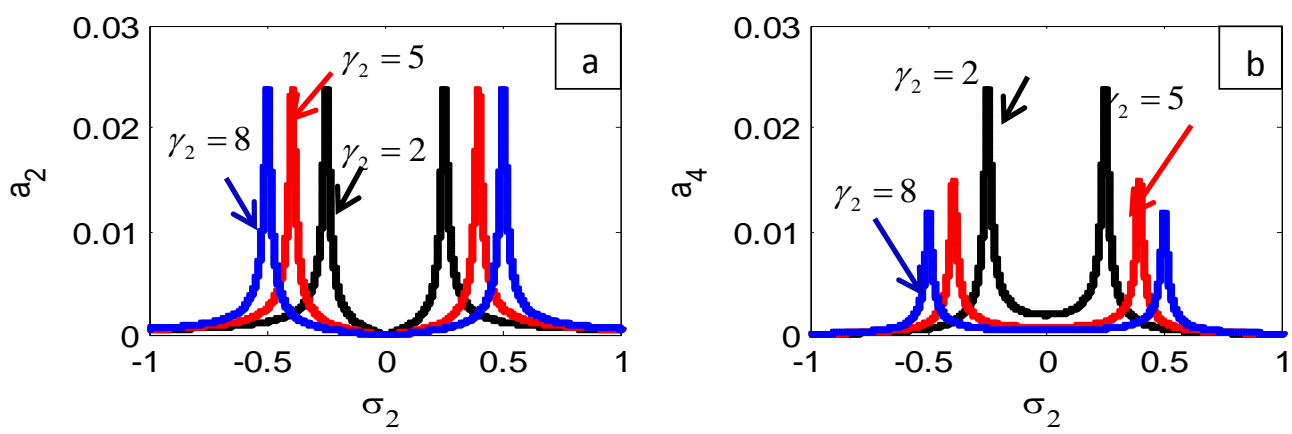

Fig 19: Influence of the control signal gain $\gamma_{2}$ (a) second mode of the system $\left(a_{2}\right)$ and (b) PPF controller $\left(a_{4}\right)$
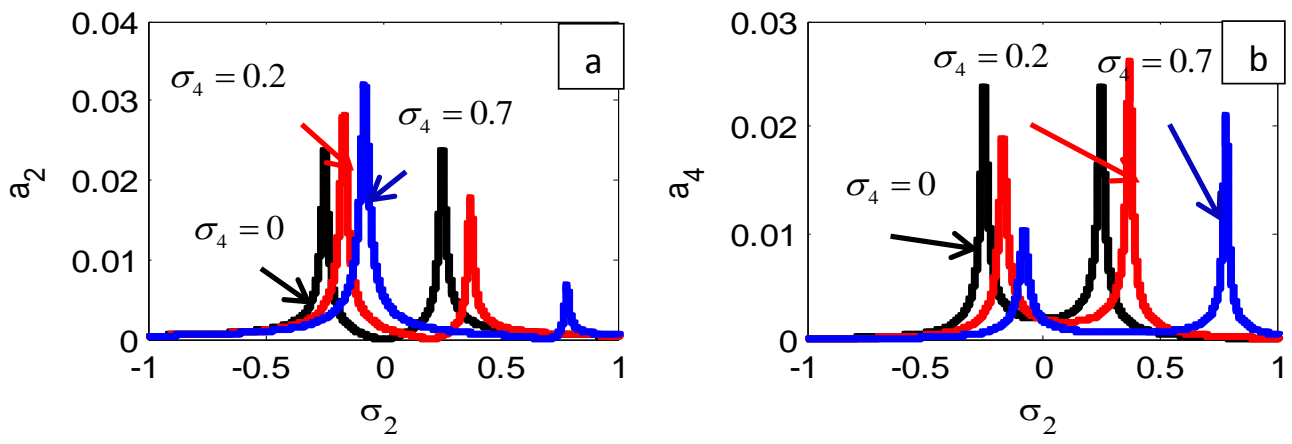

Fig 20: Influence of the detuning parameter $\sigma_{4}$ (a) second mode of the system $\left(a_{2}\right)$ and (b) PPF controller $\left(a_{4}\right)$ 


\section{Conclusions}

In this work ,the non-linear spring pendulum system simulating ship roll motion subject to external excitation force is studied. The vibration of ship roll motion can be controlled via two PPF controllers. The method of multiple scales perturbation is utilized to resolve the responses of the nonlinear system near the simultaneous primary and internal resonance, which is one of the worst cases. To discuss the stability of the system, the frequency response equations were applied. From the above study the results may be concluded.

1. Worse working conditions are obtained at simultaneous primary and internal resonance when two PPF controllers are effective. i.e. $\Omega_{1}=\omega_{1}, \Omega_{2}=\omega_{2}, \omega_{3}=\omega_{1}$, and $\omega_{4}=\omega_{2}$.

2. The amplitude of the first mode $(x)$ is reduced to about $99.92 \%$ from its value without controller and the amplitude of the second mode $(\varphi)$ is reduced to $99.99 \%$ from its value without controller.

3. The steady state amplitudes, for the practical case $\left(a_{1} \neq 0, a_{2} \neq 0, a_{3} \neq 0, a_{4} \neq 0\right)$, of the nonlinear spring pendulum are monotonic increasing functions to the excitation force amplitudes $f_{1}, f_{2}$.

4. The steady state amplitudes of the nonlinear spring pendulum are monotonic decreasing functions to the damping coefficients $c_{1}, c_{2}, \mu_{1}, \mu_{2}$.

5. The vibration reduction controller frequency bandwidth may be wider as $\lambda_{1}, \lambda_{2}, \gamma_{1}, \gamma_{2}$ are increases.

\section{REFERENCES}

1. O.A and Nayfeh, A.H. 2009 Control of Ship Roll Using Passive and Active Anti-Roll Tanks.Marzouk, Ocean Eng 36, 661-671

2. Nayfeh, A.H., Mook. 1973Nonlinear Coupled of Pitch and Roll Modes In Ship Motions . D.T. and Marshell, A.R., J. Hydronaut. 7(4), 145-152.

3. W.K. 1988 A Global Analysis of a Forced Spring-Pendulum System Lee Ph.D. Dissertation, University of California, Berkeley.

4. Song, Y., Sato, H., Iwata. Y. and Komatsuzaki, T. J. 2003. The Response of a Dynamic Vibration Absorber System with a Parametrically Excited Pendulum. Sound Vib. 259(4), 747-759 .

5. Ahamed. B. and Pota. H.R. J. 2011. Dynamic Compensation for Control of a Rotary Wing UAV Using Positive Position Feedback. Intell. Robot. Syst. 61, 43-56 .

6. Eissa, M., Kamel, M. and El-Sayed, A.T. 2010. Vibration Reduction of Multi-Parametric Excited Spring Pendulum via Transverse Tuned Absorber. Nonlinear Dyn. 61, 109-121.

7. .Eissa, M., Kamel, M. and El-Sayed, A.T. 2011. Vibration Reduction of a Nonlinear Spring Pendulum under Multi External and Parametric Excitations via a Longitudinal AbsorberMeccanica 46, 325-340 .

8. .Eissa, M., Kamel, M. and El-Sayed, A.T. 2012Vibration Suppression of a Four- Degree-Of-Freedom Nonlinear Spring Pendulum via a Longitudinal and Transverse Absorber . J. Appl. Mech. 79(1), 011007.

9. Shan, J., Liu, H. and Sun. 2005. Slewing and Vibration Control of a Single-Link Flexible Manipulator by Positive Position Feedback (PPF)., D. Mechatronics 15, 487-503.

10. El-Ganaini, W.A., Saeed, N.A. and Eissa, M. 2013. Positive Position Feedback (PPF) Controller for Suppression of Nonlinear System Vibration. Nonlinear Dyn. 72, 517-537.

11. Kwak, M.K. and Heo, S. 2007.Active Vibration Control of Smart Grid Structure by Multi Input and Multi-Output Positive Position Feedback Controller. J Sound Vibration 304, 230-45. 


\section{Author' biography with Photo}
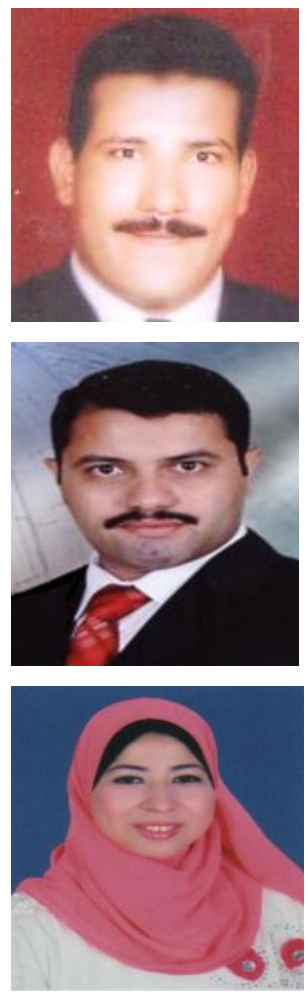

Y. A. Amer received his B.S. degree inMathematics from Zagazig University,EGYPT, in 1992. He then received his M.S.C and Ph.D. degrees from ZagazigUniversity, in 1996 and 2002, respectively. Dr. Y. A. Amer is currently a Professor of Mathematics at the Department of Mathematics, Faculty of Science, Zagaziga University, Egypt. Dr. Y. A. Amer research interests include Non-linear dynamical systems, Numerical Analysis, Vibration control and Partial differential equations.

A. T. EL-Sayed received his B.S. degree in Mathematics from Zagazig University, EGYPT, in 2001. He then received his M.S.c and Ph.D. degrees from Zagazig University, in 2007 and 2011, respectively. Dr. A. T. EL-Sayed is currently an Assistant Professor of Mathematics at the Department of Basic Sciences, Modern academy for Engineering and Technology, Egypt. Dr. A. T. EL-Sayed research interests include Differential equations which simulates non-linear dynamical systems, Numerical Analysis and Vibration control.

F. T. El-Bahrawy received her B.S. degree in Mathematics from AL-Azhar University,EGYPT, in 2005. She thenreceived her M.S.c.degrees from ALAzhar University, in 2011. F. T. El-Bahrawy is currently an Assistant Lecture of Mathematics at the Department of Basic Sciences,Modern academy for Engineering and Technology, Egypt. F. T. El-Bahrawy research interests include Differential equations, Numerical Analysis, and Vibration control. 